\title{
Complete Uterine Septum as an Incidental Finding as a Cause of Post-Partum Haemorrhage and Role of Balloon Tamponade
}

\author{
Smriti Khandelwal ${ }^{1}$, Surekha Atul Tayade², Deepti Shrivastava ${ }^{3}$, Kiran Pralhadrao Dhurve ${ }^{4}$ \\ 1, 2, 3,4 Department of Obstetrics and Gynaecology, Jawaharlal Nehru Medical College, \\ Sawangi (Meghe), Wardha, Maharashtra, India.
}

\section{INTRODUCTION}

Congenital anomalies of the uterus may cause gynaecological, obstetric and fertility problems. Mullerian duct anomalies are associated more with obstetrical complications encompassing infertility, recurrent pregnancy losses, malpresentations and poor obstetrical outcomes such as preterm delivery and rarely Post-Partum Haemorrhage (PPH). In industrialised countries there is an increase in the frequency of postpartum haemorrhage with it being the major cause of maternal mortality, also accounting for a quarter of maternal deaths worldwide. Failure of uterotonics to control bleeding, operative procedures like laparotomy with uterine, utero-ovarian or hypogastric artery ligation, uterine compression sutures such as the B-Lynch modification, or hysterectomy were considered previously but the associated morbidity and fertility preservation desire have led to usage of new therapies like balloon tamponade becoming a popular method. We are reporting a case of a young primigravida who was taken for emergency caesarean section for fetal distress and with an incidental finding of complete uterine septum as the cause of post-partum haemorrhage which was successfully managed by intrauterine balloon tamponade.

Frequently asymptomatic and unrecognised Mullerian duct anomalies account for various gynaecological, obstetrical and fertility problems commonly accounting for fetal growth restriction, increased risk of abortion, preterm labour, cervical insufficiency, abnormal presentation of fetus, hypertensive disorders of pregnancy, antepartum and postpartum haemorrhage and increased incidence of caesarean delivery. Due to wide variation in Mullerian duct anomaly presentation determining its true incidence becomes difficult. It occurs approximately in $5-10 \%$ of women with history of recurrent early pregnancy loses, $25 \%$ with first and second trimester loss or preterm labour and $3-4 \%$ of fertile and infertile women ${ }^{1}$ Septate uterus belongs to Class V Mullerian duct anomalies in which the uterus is normal on the exterior but has a partial or complete septum in the cavity. A complete or partial uterine septum occurs as a result of failure of reabsorption of the midline uterine septum between the two fused Mullerian ducts. ${ }^{2}$ Uterine septum can lead to postpartum haemorrhage either during caesarean section or following a vaginal delivery. When primary treatment to control PPH fails secondary interventions such as uterine balloon tamponade should be considered, ${ }^{1}$ which can be lifesaving. Balloon tamponade can also avoid technically demanding peripartum hysterectomy.

\section{PRESENTATION OF CASE}

A young primigravida reported to labour unit of a tertiary care center with labour pains at 40 weeks of gestation. She had a past history of infertility for which she had received treatment; however, this was a spontaneous conception.
Corresponding Author: Dr. Surekha Atul Tayade, Department of $O B G Y$, Jawaharlal Nehru Medical College, Sawangi (Meghe), Wardha, Maharashtra, India.

E-mail:surobgy@gmail.com

DOI: $10.14260 / j e m d s / 2020 / 812$

How to Cite This Article: Khandelwal S, Tayade SA, Shrivastava D, et al. Complete uterine septum as an incidental finding as a cause of post-partum haemorrhage and role of balloon tamponade. J Evolution Med Dent Sci 2020;9(48):3700-3702, $10.14260 / j e m d s / 2020 / 812$

Submission 26-05-2020,

Peer Review 14-10-2020,

Acceptance 20-10-2020,

Published 30-11-2020.

Copyright (C) 2020 JEMDS. This is an open access article distributed under Creative Commons Attribution License [Attribution 4.0 International (CC BY 4.0)] 


\section{DISCUSSION OF MANAGEMENT}

The woman had to be taken for emergency caesarean section in view of fetal distress. After the baby was extracted a longitudinal intrauterine septum was noted in the uterine cavity, extending from mid cavity to below cervix. It bled profusely and PPH bundle protocol was initiated to manage the bleeding.

The septum seemed to have separated in a leaf like manner accommodating the fetus in utero. Pressure was applied on the bleeding area to temporarily control the bleeding, after which decision was taken for bilateral uterine artery ligation which resulted in some control of the bleeding. The uterine tone was still not satisfactory, and the uterus was intermittently flabby. Uterotonics were given; however, profuse bleeding continued.

Surgeons inserted Every Second Matters - Uterine Balloon Tamponade (ESM-UBT) which is a simple device used to control PPH. The ultra-low-cost, costing less than $\$ 5$, ESM-UBT is a device consisting of a foleys catheter to which a condom is tied and inflation with clean water using a syringe is done and a one-way valve is present in it. In the United States balloon tamponade is the standard management but the US hospitals use manufactured medical balloons which are costly, costing over $\$ 400$ per unit and are of single use.

The ESM-UBT device is an ultra-affordable alternative. In the rural setting of central India, the ESM UBT was made available through a non-profit organisation and health care workers had received training for its use. In this instance the UBT proved effective in controlling the haemorrhage after instillation of $300 \mathrm{ml}$ of fluid in the condom catheter.
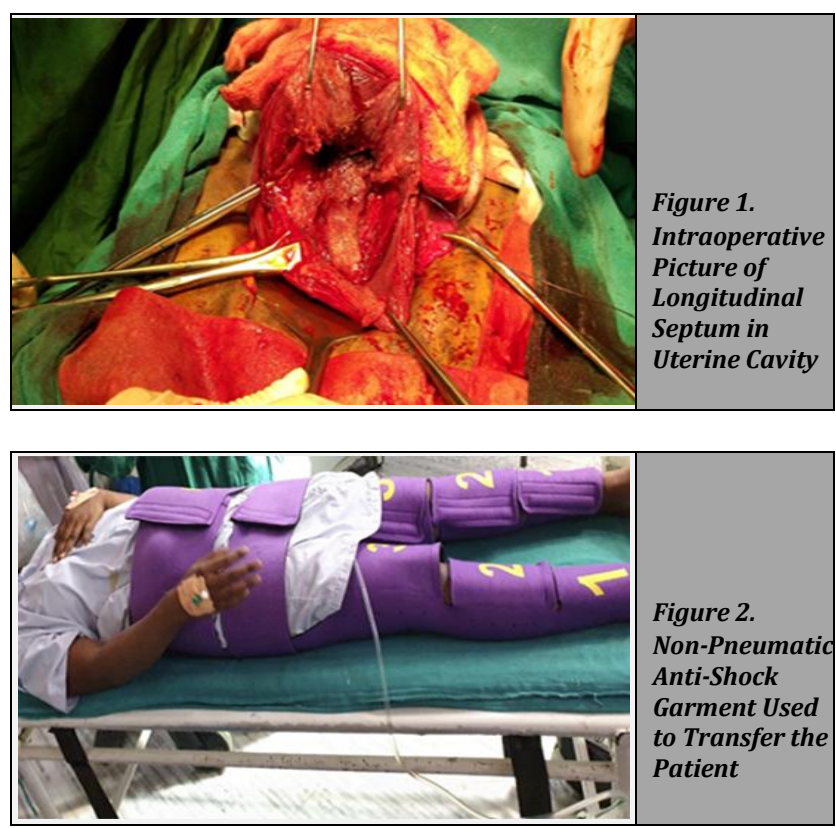

Non-Pneumatic Anti Shock Garment (NASG) was used to transfer the patient to high dependency unit for post-operative care. NASG garment was later removed systematically and the patient was stabilized haemodynamically. The balloon tamponade was kept in situ for 12 hours and then balloon deflation was done slowly removing $50 \mathrm{ml}$ per hour but after first $50 \mathrm{ml}$ removal of saline bleeding per vagina was seen again. The balloon was re-inflated till bleeding stopped and kept in situ for another 12 hours. After 24 hours balloon deflation was again attempted with simultaneous use of intravenous oxytocin in the form of infusion.

The balloon was successfully deflated over a couple of hours with no signs of bleeding per vaginum. Postpartum haemorrhage due to uterine septum was managed and controlled with help of balloon tamponade.

\section{DISCUSSION}

In the developing countries postpartum haemorrhage results in 125,000 deaths a year and accounts for around $28 \%$ of maternal mortality. Primary PPH is said to occur after $5 \%$ of deliveries but the traditional definition; blood loss $>500 \mathrm{ml}$ in the first $24 \mathrm{hrs}-$ is now recognised to be of little clinical relevance. Blood loss after delivery is notoriously difficult to measure and PPH may be best defined by a fall in haematocrit or by the need for transfusion. ${ }^{3}$ Failure of resorption of the tissue connecting the two paramesonephric (Müllerian) ducts before 20th embryonic week results in the development of uterine septum. The mildest form of this failure is the arcuate uterus which remains clinically insignificant unlike uterine septum. Since many septal defects remain asymptomatic ascertaining the true prevalence of uterine septum becomes difficult, but it appears to range between 1 to 2 per 1,000 to as high as 15 per $1,000.4$

A complete uterine septum often presents with various obstetrics complications with recurrent pregnancy loses as most common presentation, infertility, mal presentations. Rarely a complete uterine septum can present with a longitudinal lie pregnancy continuing to term with no complications. Uterine septums' are many a times an incidental finding diagnosed in patients who present with infertility. HSG (Hystero-Salpingo-Gram), hysteronlaparoscopy done as investigations for fertility help in diagnosis of septum. Undiagnosed septums' can result in bleeding after childbirth and can prove difficult to diagnose in case of vaginal birth. In the present case, the caesarean birth allowed advantage of visual identification and thorough examination.

However, associated atonicity had to be additionally managed with uterine tamponade.

Uterine tamponade has now replaced the previous method of vaginal packing to control PPH due to uterine atonicity. Dabelea et al. ${ }^{5}$ conducted a study in 2007 for the management of PPH with balloon tamponade with effectively controlling PPH in 18 cases out of 23 patients in PPH. Easy use, time saving placement with immediate results and the benefit to assess further bleeding after placing the catheter adds to the advantage of balloon tamponade. Adding balloon tamponade in PPH management protocol algorithm provides the clinician to quickly decide if morbid therapies are further required. The balloons that are used include the Sengstaken-Blakemore tube, the Bakri balloon, the Rusch balloon, Foley catheters and the condom catheter balloon. ${ }^{6}$ The intrauterine balloon is currently believed to work by mechanism of exerting inwardto-outward pressure 'that is greater than the systemic arterial pressure' preventing continuous haemorrhage. ${ }^{7}$ The hydrostatic pressure effect of the balloon on the uterine arteries is an alternative mechanism considered. ${ }^{8}$

The ESM-UBT is an ultra-low-cost, feasible, safe and highly cost-effective package designed to address the top-ranking 
cause of maternal deaths in the world. Incorporating WHO (World Health Organization) and FIGO (International Federation of Gynaecology and Obstetrics) standards for PPH management. The ESM-UBT package includes a training curriculum of 3 hours, a PPH wall poster, a job-aid checklist; a trainer's teaching flipchart; a learner's booklet; and an ESMUBT device. It very rapidly stops bleeding in uncontrolled ${ }^{P P H},{ }^{9}$ Since 2012, the WHO PPH guidelines have recommended use of the uterine balloon for uncontrolled PPH.

In this case a Mullerian anomaly remained undetected and was diagnosed as an incidental finding on caesarean section, a complete septum extending longitudinally below cervix accommodated a full term fetus with normal presentation. The anomaly did not manifest with usual complications like recurrent pregnancy loses, malpresentations but complicated postpartum with massive haemorrhage which was effectively controlled with uterine balloon tamponade.

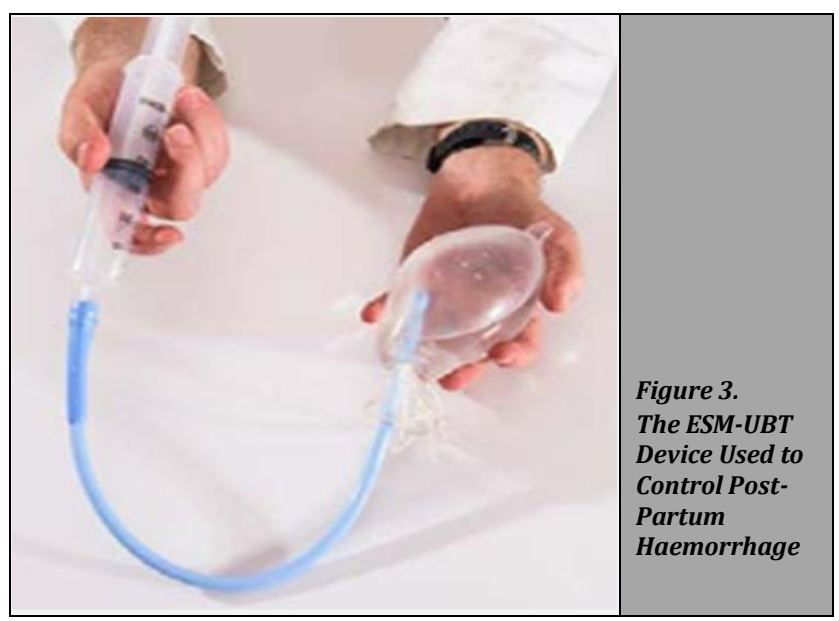

\section{CONCLUSIONS}

Mullerian anomalies may remain asymptomatic in many cases and present later with complications in pregnancy leading to their retrospective diagnosis. Though malpresentations are common with septate uterus but full-term pregnancy with longitudinal lie is no more uncommon. Uterine balloon tamponade has been successful previously to control bleeding in postpartum haemorrhage due to atonic uterus but bleeding due to atonicity as well as from septal surface could also be successfully achieved with its usage.

Financial or other competing interests: None.

Disclosure forms provided by the authors are available with the full text of this article at jemds.com.

\section{REFERENCES}

[1] Boyar IH, Boynukalın FK, Boyar N, et al. B-Lynch suture technique to control postpartum haemorrhage in a patient with mullerian anomaly. J Turk Ger Gynecol Assoc 2011;12(1):47-9.

[2] Heinonen PK. Complete septate uterus with longitudinal vaginal septum. Fertil Steril 2006;85(3):700-5.

[3] Drife J. Management of primary postpartum haemorrhage. BJOG Int J Obstet Gynaecol 1997;104(3):275-7.

[4] Pfeifer S, Butts S, Dumesic D, et al. Uterine septum: a guideline. Practice Committee of the American Society for Reproductive Medicine. Fertil Steril 2016;106(3):530-40.

[5] Dabelea V, Schultze PM, McDuffie RS Jr. Intrauterine balloon tamponade in the management of postpartum haemorrhage. Am J Perinatol 2007;24(6):359-64.

[6] Georgiou C. Balloon tamponade in the management of postpartum haemorrhage: a review. BJOG Int J Obstet Gynaecol 2009;116(6):748-57.

[7] Condous GS, Arulkumaran S, Symonds I, et al. The "tamponade test" in the management of massive postpartum haemorrhage. Obstet Gynecol 2003;101(4):767-72.

[8] Cho Y, Rizvi C, Uppal T, et al. Ultrasonographic visualization of balloon placement for uterine tamponade in massive primary postpartum haemorrhage. Ultrasound Obstet Gynecol 2008;32(5):711-3.

[9] mgh-esm-ubt-briefing.pdf [cited 2020 May 26]. https://www.elrha.org/wpcontent/uploads/2017/08/mgh-esm-ubt-briefing.pdf 